\title{
Factores de riesgo de infección de derivativa ventrículo peritoneal en pacientes pediátricos del Hospital Carlos Van Buren
}

\author{
Abigail Peña A., Rodrigo Sandia Z., Rodrigo Riveros P., Cristian Salazar Z., \\ Rosa Herrera O. y Rodrigo Vergara F.
}

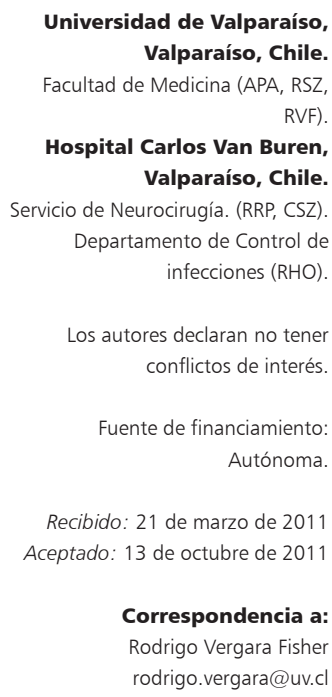

Recibido: 21 de marzo de 2011 Aceptado: 13 de octubre de 2011

Correspondencia a: Rodrigo Vergara Fisher rodrigo.vergara@uv.c

\section{Risk factors for ventricular peritoneal shunt infection in pediatric patients from the hospital Carlos Van Buren}

Hydrocephalus is a common problem in Neurosurgery and Neurology. The usual treatment is the installation of a Ventricular Peritoneal Shunt (VPS). Infection is the most frequent and serious complication. With the aim to identify risk factors associated with infection in the post surgery of VPS in pediatric patients from Carlos Van Buren Hospital a case control study between 1998-2008 was done. Cases were patients with VPS infection reported to the Department of Nosocomial Infections. Results in contingency tables were analyzed to determine Odds Ratio. 264 surgical procedures were studied in 207 patients with 53 infections reported in 26 patients. Significant risk factors were: history of prior ventriculitis, VPS dysfunction and prior external ventricular shunt, concurrent infections at the time of surgery and a neurosurgeon without the specialty of pediatric neurosurgery. We concluded that antibiotic prophylaxis was not an important factor in preventing infection and the neurosurgeon experience is relevant to the development of VPS infections.

Key words: VPS, infection, risk factors, ventriculitis, shunt infections.

Palabras clave: DVP, infección, factores de riesgo, ventriculitis, infección DVP.

\section{Introducción}

L a hidrocefalia es un problema común en neurología y neurocirugía pediátrica, cuya etiología es un desbalance entre la producción, circulación y reabsorción del líquido cefalorraquídeo $(\mathrm{LCR})^{1}$. El desarrollo de esta condición en la infancia es frecuente, siendo secundaria a un grupo variado de patologías cerebrales, entre las cuales destacan: cerebrales congénitas, traumáticas, tumorales, infecciosas y vasculares ${ }^{2}$.

Una de las opciones para el tratamiento permanente de esta patología es la inserción de un catéter junto a una válvula que conecte el ventrículo cerebral con una cavidad serosa como el peritoneo ${ }^{3}$ o derivativa ventrículo peritoneal (DVP) o con una vena profunda, donde el exceso de LCR pueda ser drenado.

Se estima que entre 25 y $46 \%$ de las derivaciones tienen un mal funcionamiento durante el primer año después de haber sido instaladas ${ }^{4,5}$, siendo la obstrucción mecánica y las infecciones, las dos principales causas de disfunción valvular $^{6,7}$.

La tasa de infección post instalación de DVP en la población infantil se estima en $10 \%$ y fluctúa entre
0 y $22 \%{ }^{8-11}$, siendo una causa importante de morbimortalidad $^{12}$. Las infecciones tempranas son causadas por la microbiota bacteriana introducida al momento de la cirugía, mientras que las tardías corresponden a contaminaciones posteriores ${ }^{13}$.

Se han identificado diversos factores del paciente y de la atención asociados a una infección de DVP ${ }^{14}$. Entre los factores relacionados con el paciente destacan: prematurez ${ }^{15}$, edad menor de cuatro meses, diagnóstico antenatal de hidrocefalia ${ }^{16}$, enfermedad sistémica concomitante ${ }^{17}$, requerimientos previos de derivativa ventricular externa (DVE), estado de conciencia alterado y uso de terapia oncológica adyuvante (quimioterapia y radioterapia) ${ }^{15}$.

También debemos considerar dentro de este grupo la etiología de la hidrocefalia, la cual pudiese llegar a ser un factor predictor en sí mismo de la infección de derivativa. De hecho, la hemorragia intraventricular o intracerebral (HIC) $)^{9,16,17}$, la hemorragia subaracnoídea ${ }^{17}$ y el mielomeningocele ${ }^{16}$, han mostrado tener una mayor tasa de infecciones que otras patologías que requieren de derivación ${ }^{18}$.

Respecto a los factores relacionados con la atención en salud, se cuentan: una deficiente técnica quirúrgica ${ }^{19}$, 
tiempos operatorios prolongados ${ }^{15,20}$ y el rasurado de la piel a intervenir ${ }^{21,22}$.

Otros factores neuroquirúrgicos identificados son: presencia de una fístula de LCR, craneotomía, hipertensión endocraneana (presión intracraneal > $20 \mathrm{mmHg}$ ), ventriculostomía de duración prolongada (particularmente en los primeros 7 a 10 días), irrigación del sistema y recambio valvular a repetición ${ }^{9,17}$.

A pesar de lo anteriormente señalado, actualmente todavía falta conocimiento acerca de los factores de riesgo para infección de DVP, dado que la información publicada es muy variada y muchas veces contradictoria, careciendo aún de estudios con alto nivel de evidencia que corroboren los hallazgos de estudios retrospectivos. Además, a la fecha no encontramos información originada en el medio nacional.

En el Hospital Carlos van Buren de Valparaíso (HCVB) no existe evidencia demostrada respecto a los factores determinantes de infecciones de las DVPs. El objetivo principal de este trabajo es identificar en este centro aquellos factores de riesgo asociados a la infección de las derivativas, tanto dependientes del paciente como de la atención. Secundariamente, pretendemos determinar las tasas de incidencia de infección de DVP, determinar el perfil microbiológico de las infecciones y conocer la susceptibilidad antimicrobiana de los microorganismos aislados.

Finalmente, deseamos aportar en el avance de la prevención de infecciones en dicho centro hospitalario, contribuyendo además con esta experiencia a la realización de nuevos estudios en otros hospitales, que permitan conocer más a cabalidad los factores involucrados en la infección de DVP.

\section{Material y Método}

Se realizó un estudio observacional, retrospectivo, de casos y controles, en que los participantes fueron todos los niños con hidrocefalia entre 0 y 15 años, y que se les hubiese instalado una válvula DVP entre enero de 1998 y junio de 2008 en el HCVB de Valparaíso.

Los pacientes estudiados fueron identificados según la revisión de todos los protocolos de cirugías del Servicio de Neurocirugía del HCVB en el plazo ya mencionado. Cada instalación de derivativa se consideró como un evento de estudio, ya que algunos pacientes contaban con más de una intervención registrada.

\section{Definición de infección de DVP}

Diagnóstico médico hecho en aquellos pacientes con parámetros clínicos compatibles (cefalea, vómitos, aumento del perímetro craneano), parámetros de laboratorio alterados (leucocitosis $>12.000$ céls $/ \mathrm{mm}^{3}, \mathrm{PCR}>6 \mathrm{mg} / \mathrm{L}$ ) $\mathrm{y} / \mathrm{o}$ con signos imagenológicos indirectos a la tomografía axial computada de cerebro (aumento del tamaño ventri- cular respecto a imágenes previas), cuya confirmación se realiza a través del análisis de LCR obtenido desde una punción lumbar, exploración intra-operatoria o punción del reservorio de la DVP.

Para identificar las infecciones de DVP (los llamados "casos" o "eventos") se revisaron los registros del Departamento de Epidemiología e Infecciones Intrahospitalarias del HCVB, donde la notificación de infección de DVP se hace solamente una vez (al primer evento) en cada paciente, sin importar que presente infecciones posteriores de la misma causa.

Los eventos infecciosos posteriores en los pacientes se identificaron por la revisión de fichas clínicas, sin hacer distinción de la forma en la cual se obtuvo el diagnóstico (clínica, imágenes, laboratorio o análisis de LCR).

Se utilizó como criterio de exclusión de casos, que el paciente hubiera presentado infección de su DVP después de un año de la instalación de la misma. Según este criterio no hubo pacientes excluidos. Además fueron excluidos aquellos pacientes con notificación de infección pero cuya ficha clínica no estuviera disponible impidiendo un análisis del caso.

Se determinó elegir mediante azar simple, para cada paciente notificado, el evento infeccioso que sería incorporado al estudio, con el fin de considerar sólo un evento de infección valvular por paciente.

Se decidió arbitrariamente que el número de controles serían dos por cada caso, para lo cual se calculó en forma proporcional por año, el número de eventos no infecciosos equivalentes a las infecciones presentadas en ese mismo lapso de tiempo. Para obtener los controles que entrarían al estudio, se seleccionaron en forma aleatoria los pacientes anualmente hasta obtener el número de eventos necesarios previamente calculado para el período estudiado.

Se calculó la incidencia anual de la infección de DVP, sin estudiar posibles causas de variación de la incidencia en los distintos períodos.

Las variables consideradas en el estudio se ordenaron en dos grupos:

- Factores asociados al paciente, entre los cuales estuvieron la edad, sexo, la causa de hidrocefalia, ventriculitis previa, antecedente de disfunción de DVP, siendo estas dos últimas variables consideradas sólo si ocurrieron dentro del mismo año de la infección de DVP registrada; además se consideraron los antecedentes de infecciones concomitantes graves (sepsis, infección del tracto urinario (ITU), meningitis, neumonía) que el paciente hubiera presentado dentro del mismo período de hospitalización que precedió a la instalación de su DVP (sin evaluar la similitud etiológica de la infección concomitante con la infección de DVP), y el número de DVP y DVE instaladas sin un límite de tiempo en relación al evento de DVP estudiado.

- Factores asociados a la atención, donde se consideraron 
variables asociadas al procedimiento quirúrgico: oportunidad de profilaxis antimicrobiana (administración del fármaco, 30 minutos a una hora previa al inicio de la cirugía), tipo de antiséptico intra-operatorio, profesional tratante que realizó el procedimiento (distinguiendo entre neurocirujanos con especialización certificada en neurocirugía pediátrica y los que no la tienen), tipo de cirugía realizada (programada o de urgencia), pabellón en el cual se realizó la intervención (pabellón neuroquirúrgico o de urgencia), realización de craneotomía durante la operación y tiempo empleado en la cirugía.

\begin{tabular}{|c|c|c|c|c|}
\hline & $\begin{array}{c}\text { Total n (\%) } \\
78\end{array}$ & $\begin{array}{c}\text { Casos n (\%) } \\
26\end{array}$ & $\begin{array}{c}\text { Controles n (\%) } \\
52\end{array}$ & valor $\mathbf{p}$ \\
\hline \multicolumn{5}{|l|}{ Sexo } \\
\hline Femenino & $34 / 78(43,6)$ & $15 / 26(57,7)$ & $19 / 52(36,5)$ & $N S^{*}$ \\
\hline Edad (meses) & 3,7 (mediana) & 4,9 (mediana) & 3,7 (mediana) & $\mathrm{NS}^{+}$ \\
\hline \multicolumn{5}{|c|}{ Causa de hidrocefalia } \\
\hline $\mathrm{HIC}$ & $25 / 78(32)$ & $11 / 26(42,3)$ & $14 / 52(26,9)$ & $N S^{*}$ \\
\hline MMC & $4 / 78 \quad(5,1)$ & $1 / 26 \quad(3,8)$ & $3 / 52 \quad(5,8)$ & $N S^{*}$ \\
\hline meningitis & $6 / 78 \quad(7,7)$ & $3 / 26(11,5)$ & $3 / 52 \quad(5,8)$ & $N S^{*}$ \\
\hline malformación & $6 / 78 \quad(7,7)$ & $2 / 26 \quad(7,7)$ & $4 / 52 \quad(7,7)$ & $N S^{*}$ \\
\hline tumor & $21 / 78(26,9)$ & $6 / 26(23)$ & $15 / 52(28,8)$ & $N S^{*}$ \\
\hline congénita & $1 / 78 \quad(1,3)$ & 0 & $1 / 52 \quad(1,9)$ & $N S^{*}$ \\
\hline TEC & $1 / 78 \quad(1,3)$ & 0 & $1 / 52 \quad(1,9)$ & $N S^{*}$ \\
\hline sepsis & $5 / 78 \quad(6,4)$ & $1 / 26 \quad(3,8)$ & $4 / 52 \quad(7,7)$ & $N S^{*}$ \\
\hline
\end{tabular}

NS: No significativo HIC: hemoragia intracraneal. MMC: mielomeningocele. TEC: traumatismo encéfalocraneal. *Prueba de $\chi^{2}$. 'Prueba de Mann Whitney.

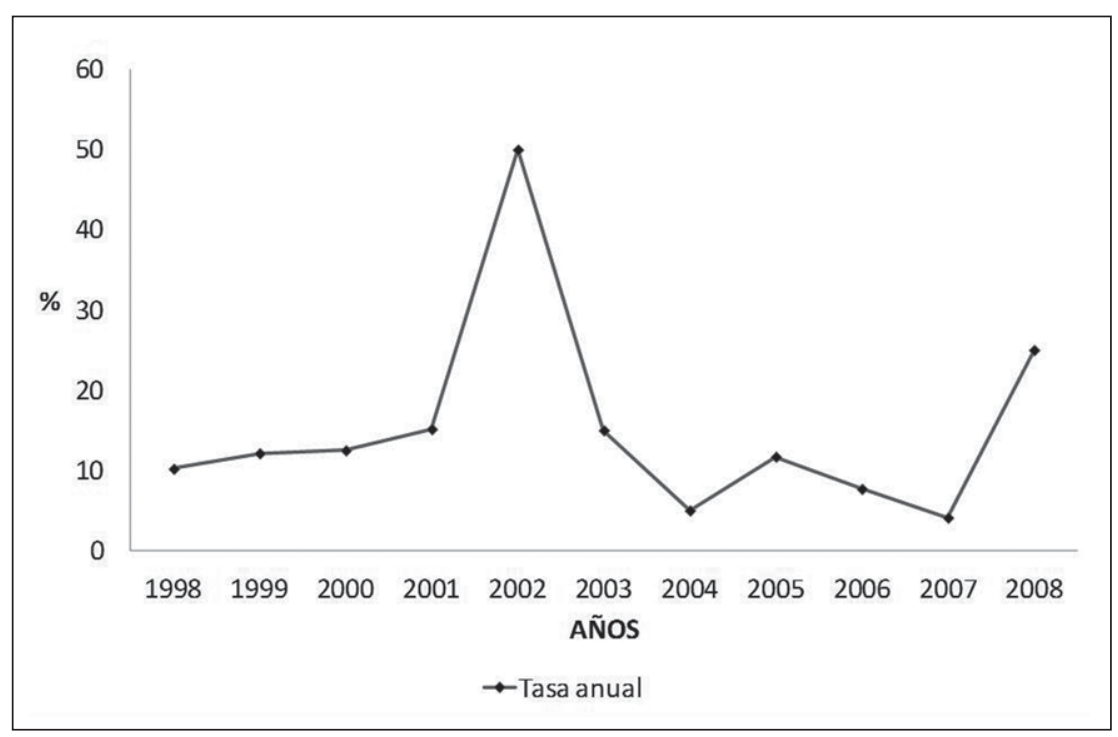

Figura 1. Incidencia anual de infección de DVP.
Para la obtención de los datos se procedió a la revisión de 78 fichas clínicas en el archivo central del HCVB, previa autorización de las autoridades correspondientes.

El análisis estadístico fue realizado íntegramente en el programa SPSS 11.5 ® para Windows. Se comparó la presencia o ausencia de los distintos factores de riesgo en relación al posterior desarrollo de infección de la DVP separando las variables estudiadas en dos grupos: casos y controles.

Los datos fueron comparados con las prueba de Mann Whitney y $\chi^{2}$ según correspondiera. Además fueron calculados los odds ratios (OR) con un intervalo de confianza (IC) del 95\% de las variables consideradas como factores de riesgo. Para la estadística descriptiva del total de pacientes (Tabla 1), los datos fueron descritos en medianas y frecuencias absolutas y relativas (porcentajes). Se consideró como significativos valores de p menores a 0,05 e IC 95\% que no incluyeran el uno.

Para analizar el OR del neurocirujano (NC) que realizó el procedimiento, agrupamos esta variable de tres categorías (NC adulto, NC infantil y becado de neurocirugía) en dos categorías, generando así una nueva variable dicotómica. En la primera categoría estuvieron agrupados dos variables (ejemplo: NC infantil y becados) y en la segunda categoría solamente se consideró una variable (ejemplo: $\mathrm{NC}$ adulto). De esta forma se midió el OR de infección de DVP de un cirujano respecto al riesgo de los otros dos cirujanos. Cabe destacar que del total de procedimientos quirúrgicos efectuados por el grupo considerado dentro de los becados de neurocirugía, $85 \%$ fue supervisado por un NC infantil, y del 15\% restante de las intervenciones no se pudo obtener un registro.

Las medidas básicas de prevención de infecciones (lavado de manos, uso de guantes, utilización de materiales y equipo estéril, etc.) fueron las mismas tanto para casos como para controles, por lo que no se consideraron en nuestra investigación.

\section{Resultados}

En el período estudiado se registraron 264 instalaciones de DVP en 207 pacientes, con un total de 34 pacientes notificados con infección. Hubo una pérdida de ocho pacientes infectados debido a que no se pudo acceder a la ficha clínica ni a otro tipo de registros médicos. La incidencia de infección fue variable en los distintos años de estudio, con un mínimo de 4,1\% en 2007 y un máximo de $50 \%$ en 2002 (Figura 1).

En los 26 pacientes notificados de los que sí se pudo obtener historial médico, se presentaron 53 eventos infecciosos de DVP. Las características generales de los casos y controles se muestran en la Tabla 1, donde se observa que entre los grupos no hubo diferencias significativas en cuanto a edad, sexo y causa de hidrocefalia. 
Respecto a los microorganismos causantes de la infección de la DVP, 13 de las 26 infecciones fueron causadas por Staphylococcus coagulasa negativa, seguido por seis infecciones por Staphylococcus aureus y luego por Klebsiella spp, Escherichia coli, Streptococcus grupo viridans y Pseudomonas spp, cada uno de ellas responsable de un caso. Hubo tres casos en los que no se obtuvo información.

Con respecto al patrón de susceptibilidad, si bien varios de los registros tenían información incompleta, se pudo determinar que de los 13 Staphylococcus coagulasa negativa, ocho fueron resistentes a meticilina (cloxacilina) y sensibles a vancomicina, uno sensible a vancomicina y cotrimoxazol, dos fueron sensibles a cloxacilina y en dos casos no se obtuvo registro de susceptibilidad.

Dos aislados de $S$. aureus fueron informados resistentes a meticilina y sensibles a vancomicina, dos sensibles a cloxacilina y en dos casos no se obtuvo registro de susceptibilidad.

Las cepas de Klebsiella spp y E. coli fueron sensibles a cefalosporinas de tercera generación y a aminoglucósidos mientras que la cepa de Streptococcus grupo viridans solamente era sensible a vancomicina.

Del aislado de Pseudomonas sp, no se pudo obtener el registro de susceptibilidad.

Las variables que mostraron significativamente ser factores de riesgo para la infección de DVP fueron: el antecedente de disfunción valvular, ventriculitis previa, presencia de infecciones sistémicas concomitantes al momento de la instalación de la derivación y cirugía realizada por un neurocirujano sin especialización en neurocirugía pediátrica.

El uso de profilaxis antimicrobiana con cefazolina (30 a $50 \mathrm{mg}$ por $\mathrm{kg}$ de peso) al inicio del procedimiento anestésico no mostró ser un factor protector. No hubo variaciones en la profilaxis efectuada en casos y en controles, como tampoco tratamientos profilácticos intensificados en ninguno de los dos grupos.

No se encontraron diferencias significativas entre los tiempos operatorios de los casos y los controles, así como tampoco se encontraron diferencias en el pabellón donde se hizo la cirugía (central o de urgencia), el tipo de cirugía (electiva o de urgencia), la realización de craneotomía y el tipo de antiséptico utilizado.

En este último aspecto, cabe destacar que el antiséptico utilizado en los procedimientos quirúrgicos durante el período de estudio correspondió a povidona yodada al $10 \%$, exceptuando en los procedimientos realizados por los especialistas en neurocirugía pediátrica, donde en la totalidad de las oportunidades el antiséptico utilizado fue clorhexidina gluconato $0,5 \%$.

Otras variables de interés, así como el detalle de algunas de las variables antes señaladas, se muestran en la Tabla 2.

Los casos mostraron un historial de mayor número de
Tabla 2. Factores de riesgo de infección de derivativa ventrículo-peritoneal

\begin{tabular}{|lccccc|}
\hline & Casos & Controles & OR & $\begin{array}{c}\text { IC 95\% } \\
\text { Límite }\end{array}$ & $\begin{array}{c}\text { Límite } \\
\text { inferior }\end{array}$ \\
superior \\
Profilaxis antimicrobiana & 17 & 36 & 0,8 & 0,30 & 2,28 \\
Ventriculitis previa & 18 & 19 & 3,9 & 1,42 & 10,68 \\
Infecciones concomitantes & 15 & 14 & 3,7 & 1,37 & 9,96 \\
Disfunción DVP previa & 14 & 5 & 10,9 & 3,29 & 36,47 \\
Neurocirujano pediátrico & 9 & 26 & 0,5 & 0,20 & 1,40 \\
Neurocirujano adulto & 17 & 26 & 2,8 & 1,04 & 7,80 \\
Craneotomía & 8 & 20 & 0,7 & 0,26 & 1,93 \\
Clorhexidina 0,5\% & 9 & 26 & 0,5 & 0,20 & 1,40 \\
\hline *Infecciones concomitantes: sepsis de cualquier foco, neumonía, infección urinaria, meningitis. \\
\hline
\end{tabular}

recambios tanto de DVP (mediana de 1 versus 0 ) como de DVE (mediana de 2 versus 0 ) respecto a los controles; ambas diferencias mostraron ser estadísticamente significativas $(\mathrm{p}<0,001)$. Como dato anexo, 19 de los casos (73\%) y 36 de los controles $(69,2 \%)$ tenían antecedentes de DVE previas, y el antecedente de DVP estuvo presente en 15 casos $(57,7 \%)$ y 22 controles $(42,3 \%)$.

\section{Discusión}

Hasta donde sabemos, este es el primer estudio de factores de riesgo de ventriculitis asociada a DVP en Chile. La incidencia de ventriculitis asociada a DVP en nuestro centro es similar a lo encontrado en otros reportes, en los que fluctúa entre 4 y $15 \%{ }^{1,23}$. Si bien es cierto que en algunos períodos como 2002 y 2008 esta tasa se elevó, llegando hasta valores de $50 \%$, no pudimos encontrar causales válidas que explicaran los resultados obtenidos, porque no hay una evidencia concreta que muestre diferencias técnicas, humanas o instrumentales que pudiesen explicar esta alza.

Un punto controversial dentro de nuestra metodología es la selección al azar de un evento infeccioso por paciente. Quizás el haber considerado únicamente el primer evento infeccioso pudiera haber establecido de una manera más pura los resultados respecto a los factores de riesgo, pero quisimos con nuestra medida, representar de una mejor manera a la población pediátrica de nuestro centro, que generalmente tiene antecedentes de DVP o DVE instaladas previamente al episodio infeccioso de la DVP en particular. Por otra parte, nuestro razonamiento también se basa en que muchas veces existe un período de tiempo distante (más de un año) entre la infección de DVP estudiada y las DVP o DVE infectadas previamente, por lo 
que es conveniente considerarlas como sucesos independientes. En otro sentido, y pese a no ser claramente factor de riesgo, los casos presentaron mayor número de recambios de DVE y DVP, lo que sugiere que la probabilidad de infecciones de las DVP aumenta proporcionalmente al número de procedimientos invasores a los que el paciente haya sido expuesto. Sin embargo, nuestro trabajo no pudo establecer una correlación temporal entre estos eventos, por lo que no sería prudente establecer conclusiones.

Entre los factores relevantes a considerar se encuentra la presencia de infecciones concomitantes, que constituyeron un importante factor de riesgo para infección de DVP. Sin embargo, una importante debilidad de nuestra metodología, es la inexistencia de la correlación microbiológica entre estas infecciones concomitantes y el agente aislado en la infección de DVP, ya que no se puede asumir a priori una causa directa y única entre los focos concomitantes de infección.

La profilaxis antimicrobiana mostró una discreta tendencia favorable en la prevención de las infecciones en pacientes con DVP, pero sin una significancia estadística. Con respecto a este punto, los resultados y las recomendaciones en la literatura médica también son controversiales ${ }^{24}$ y con beneficios no establecidos, a pesar de lo cual algunos autores siguen recomendando su uso ${ }^{25,26}$. Sería interesante en este sentido complementar nuestros hallazgos con una investigación con profilaxis diferenciadas, que no tan sólo se base en la oportunidad, sino que considere el microorganismo específico a cubrir en la eventualidad de que los pacientes tengan el antecedente de un cultivo o infecciones previas por microorganismos multi-resistentes, lo cual creemos ayudaría a establecer de una mejor manera la utilidad de la profilaxis en las infecciones de DVP.

Dentro de los factores de la atención se identificó como elemento de riesgo la intervención realizada por un neurocirujano sin especialización pediátrica (e inversamente apareció como protector -aunque sin significancia estadística- la intervención realizada por un neurocirujano pediátrico). Es probable que el número de intervenciones realizadas por uno y otro implique diferencias en la técnica quirúrgica que pudieran resultar claves en el riesgo diferencial de infecciones. Esto último se condice con lo reportado en otros centros, donde también se considera la experiencia del neurocirujano como un factor importante en la infección de $\mathrm{DVP}^{19}$. Como dato adicional, $36 \%$ de las cirugías de revisiones de derivativas (evaluación del funcionamiento del shunt después de que éste ha sido instalado) son potencialmente evitables con una mejor técnica quirúrgica ${ }^{19}$. En algunos estudios científicos internacionales se han encontrado más fracasos y aparición de complicaciones después de intervenciones realizadas por los médicos becados o por neurocirujanos con menos años de ejercicio. De esto, podría inferirse que la tasa de infecciones de DVP aumenta cuando el procedimiento es realizado por un facultativo con poca experiencia. No quisimos establecer tasas o riesgos individuales por cirujano dentro de nuestra investigación, para evitar hacer críticas o establecer distinciones personales al respecto; sin embargo, es prudente recalcar que una óptima técnica quirúrgica y realizada por un facultativo calificado, en este caso un neurocirujano pediátrico, pareciera ser razonable en las estrategias para reducir la frecuencia de revisiones de derivativas ${ }^{19}$. Otro dato a considerar es el hecho de que los facultativos en neurocirugía pediátrica utilicen otro tipo de antiséptico en relación a los otros neurocirujanos. Si bien es cierto que el uso de clorhexidina no mostró ser un factor protector en sí, pudiera haber una tendencia a la disminución de las infecciones de DVP con su utilización, por lo que en nuestro centro hospitalario puede ser recomendable establecer protocolos que indiquen y fomenten esta práctica.

Esta investigación aparece como el primer reporte de un centro neuro-quirúrgico nacional y regional, respecto a factores de riesgo para infección de pacientes portadores de DVP. Tiene la limitación de ser retrospectivo, haciéndose necesario, idealmente, una cohorte multicéntrica, que confirme los hallazgos reportados para así plantear estrategias racionales en la prevención de estas complicaciones postquirúrgicas. Además, por el hecho de haber obtenido la información y los datos del estudio a través de la revisión de fichas clínicas, siempre existe la posibilidad de la subnotificación o de una mala interpretación de los datos.

Pese a todas las limitaciones existentes en nuestro estudio y a la posible pérdida de información que pudiese haber alterado alguno de nuestros resultados, parece razonable insistir en seleccionar adecuadamente el momento de la intervención quirúrgica basado en adecuados criterios clínicos infectológicos. Además, todas las intervenciones en forma ideal, debieran ser realizadas por un operador habituado a este procedimiento y con especialización en neurocirugía pediátrica.

\section{Resumen}

La hidrocefalia es un problema común en neurocirugía y neurología. Su tratamiento habitual es la instalación de una válvula derivativa ventrículo peritoneal (DVP) cuya complicación más grave y frecuente es la infección. Con el propósito de identificar los factores de riesgo de infección post-operatoria en pacientes con DVP del Hospital Carlos Van Buren (HCVB), se realizó un estudio caso-control en la población pediátrica con DVP instalada entre 1998 y 2008. Los casos fueron pacientes con una infección de DVP notificada en el Departamento de Infecciones 
Intrahospitalarias (IIH), y controles los que no presentaron infección. Se analizaron los resultados en tablas de contingencia para determinar los Odds Ratio correspondientes. Se estudiaron 264 procedimientos quirúrgicos, 207 pacientes y 53 infecciones notificadas en 26 enfermos. Los factores de riesgo significativos fueron el antecedente de ventriculitis previa, disfunción de DVP previa, derivativa ventricular externa previas, infecciones concomitantes al momento de la cirugía, y que el neurocirujano no tuviera la especialidad en neurocirugía pediátrica. Se concluyó que la profilaxis antimicrobiana no fue un factor importante en la prevención de infecciones y que la experiencia del neurocirujano es relevante en el desarrollo de infecciones de DVP.

\section{Referencias}

1.- Turgut M, Alabaz D, Erbey F, Kocabas E, Erman T, Alhan E, et al. Cerebrospinal fluid shunt infections in children. Pediatr Neurosurg 2005; 41: 131-6.

2.- Kulkarni A V, Drake J M, Lamberti-Pasculli M. Cerebrospinal fluid shunt infection: a prospective study of risk factors. J Neurosurg 2001; 94: 195-201.

3.- Vinchon M, Baroncini M, Laurent T, Patrick D. Bowel perforation caused by peritoneal shunt catheters: diagnosis and treatment. Neurosurgery 2006; 58: 76-82.

4.- Caldarelli M, Di Rocco C, La Marca F. Shunt complications in the first postoperative year in children with meningomyelocele. Childs Nerv Syst 1996; 12: 748-54.

5.- Kim T Y, Stewart G, Voth M, Moynihan J A, Brown L. Signs and symptoms of cerebrospinal fluid shunt malfunction in the pediatric emergency department. Pediatr Emerg Care 2006; 22: 28-34

6.- Blount J P, Campbell J A, Haines S J. Complications in ventricular cerebrospinal fluid shunting. Neurosurg Clin N Am 1993; 4: 633-56.

7.- Lee J Y, Wang K C, Cho B K. Functioning periods and complications of 246 cerebrospinal fluid shunting procedures in 208 children. J Korean Med Sci 1995; 10: 275-80.

8.- Aucoin P J, Kotilainen H R, Gantz N M, Davidson R, Kellogg P, Stone B. Intracranial pressure monitors. Epidemiologic study of risk factors and infections. Am J Med 1986; 80: 369-76.
9. Mayhall C G, Archer N H, Lamb V A, Spadora A C, Baggett J W, Ward J D, et al. Ventriculostomy-related infections. A prospective epidemiologic study. N Engl J Med 1984; 310: 553-9.

10.- Stenager E, Gerner-Smidt P, Kock-Jensen C. Ventriculostomy-related infections--an epidemiological study. Acta Neurochir (Wien) 1986; 83: 20-3.

11.- Thompson D N, Hartley J C, Hayward R D. Shunt infection: is there a near-miss scenario? J Neurosurg 2007; 106: 15-9.

12.- Tuli S, Drake J, Lawless J, Wigg M, Lamberti-Pasculli M. Risk factors for repeated cerebrospinal shunt failures in pediatric patients with hydrocephalus. J Neurosurg 2000; 92: 31-8.

13.- Baird C, O'Connor D, Pittman T. Late shunt infections. Pediatr Neurosurg 1999; 31: 269-73.

14.- Simon T D, Hall M, Riva-Cambrin J, Alberg J E, Jeffries H E, LaFleur B, et al. Infection rates following initial cerebrospinal fluid shunt placement across pediatric hospitals in the United States. Clinical article. J Neurosurg Pediatr 2009; 4: 156-65.

15.- Ritz R, Roser F, Morgalla M, Dietz K, Tatagiba M, Will B E. Do antibioticimpregnated shunts in hydrocephalus therapy reduce the risk of infection? An observational study in 258 patients. BMC Infect Dis 2007; $7: 38$.

16.- Vinchon M, Dhellemmes P. Cerebrospinal fluid shunt infection: risk factors and long-term follow-up. Childs Nerv Syst 2006; 22: 692-7.

17.- Lozier A P, Sciacca R R, Romagnoli M F, Connolly E S Jr. Ventriculostomy-related infections: a critical review of the literature. Neurosurgery 2002; 51: 170-81.

18.- McGirt M J, Leveque J C, Wellons J C $3^{\text {rd }}$, Villavicencio A T, Hopkins J S, Fuchs H E, et al. Cerebrospinal fluid shunt survival and etiology of failures: a seven-year institutional experience. Pediatr Neurosurg 2002; 36: 248-55.

19.- Enger P O, Svendsen F, Sommerfelt K, Wester K. Shunt revisions in children-can they be avoided? Experiences from a populationbased study. Pediatr Neurosurg 2005; 41: 300-4.

20.- National Nosocomial Infections Surveillance (NNIS) System Report. 2004 en http://www.cdc. gov/ncidod/dhqp/pdf/nnis/2004NNISreport.pdf (accedido: 25 agosto 2009).

21.- Alexander J W, Fischer J E, Boyajian M, Palmquist J, Morris M J. The influence of hair-removal methods on wound infections. Arch Surg 1983; 118: 347-52.

22.- Ratanalert S, Saehaeng S, Sripairojkul B, Liewchanpattana K, Phuenpathom N. Nonshaved cranial neurosurgery. Surg Neurol 1999; 51: 458-63.

23.- Livni G, Yuhas Y, Ashkenazi S, Michowiz S. In vitro bacterial adherence to ventriculoperitoneal shunts. Pediatr Neurosurg 2004; 40: 64-9.

24.- Camboulives J, Meyrieux V, Lena G. Infections of cerebrospinal fluid shunts in the child: prevention and treatment. Ann Fr Anesth Reanim 2002; 21: 84-9.

25.- Kanev P M, Sheehan J M. Reflections on shunt infection. Pediatr Neurosurg 2003; 39: 285-90.

26.- Filka J, Huttova M, Tuharsky J, Sagat T, Kralinsky K, Krcmery V. Nosocomial meningitis in children after ventriculoperitoneal shunt insertion. Acta Paediatr 1999; 88: 576-8. 